\title{
Minimal clinically important difference of 3-minute chair rise test and the DIRECT questionnaire after pulmonary rehabilitation in COPD patients
}

This article was published in the following Dove Medical Press journal: International Journal of COPD

Jonathan Lévesque,' Anestis

Antoniadis, ${ }^{2}$ Pei Zhi Li, ${ }^{3}$

Frédéric Herengt, ${ }^{4}$ Christophe

Brosson, ${ }^{5}$ Jean-Marie

Grosbois, ${ }^{6}$ Alain Bernady, ${ }^{7}$

Anthony Bender, 8,9 Murielle

Favre, ${ }^{10}$ Antoine Guerder, ${ }^{11}$

Pascale Surpas, ${ }^{12}$ Thomas

Similowski, ${ }^{11,13}$ Bernard

Aguilaniu ${ }^{14}$

'Department of Medicine, Pneumology Service, Hôpital Maisonneuve-Rosemont, Faculty of Medicine, Université de Montréal, Montreal, Quebec, Canada; ${ }^{2}$ Laboratoire Jean Kuntzmann UMR5224, Statistics Department, Université Grenoble-Alpes, Grenoble, France; ${ }^{3}$ Montreal Chest Institute, McGill University Health Center Research Institute, McGill University, Montreal, Quebec, Canada; ${ }^{4}$ Centre de Réadaptation Cardio-RespiratoireDieulefit Santé, Dieulefit, France; ${ }^{5}$ Private Physiotherapist, Cours Liberation, Grenoble, France; 'Pneumology Service, Centre Hospitalier de Béthune, Béthune, France; ${ }^{7}$ Toki-Eder Centre Médical CardioRespiratoire, Cambo-les-Bains, France; ${ }^{8}$ Private Physiotherapist, Boulevard de Metz Mont-Saint-Martin, France; ' $L U N E X$ International University of Health, Exercise and Sports, Differdange, Luxembourg; ${ }^{10}$ Centre de Pneumologie Henri Bazire, Saint-Julien-de-Ratz, France; "'AP-HP, Groupe Hospitalier Pitié-Salpêtrière Charles Foix, Service de Pneumologie, Médecine Intensive et Réanimation, Département R3S, Paris, France;

${ }^{12}$ Centre Médical de Bayère, Charnay, France; ${ }^{13}$ Sorbonne Université, INSERM, UMRSII 58 Neurophysiologie Respiratoire Expérimentale et Clinique, Paris, France; ${ }^{14}$ Faculty of Medicine, Université GrenobleAlpes, Grenoble, France

Correspondence: Bernard Aguilaniu Université Grenoble-Alpes, 14 Rue Jean BOCQ, Grenoble 38000, France

Email b.aguilaniu@icloud.com
Background: The 3-minute chair rise test (3-minute CRT) and the Disability Related to COPD Tool (DIRECT) are two reproducible and valid short tests that can assess the benefit of pulmonary rehabilitation (PR) in terms of functional capacity and dyspnea in everyday activities. Methods: We determined the minimal clinically important difference (MCID) of the DIRECT questionnaire and 3-minute CRT using distribution methods and anchor encroaches with a panel of eight standard tests in a cohort of 116 COPD patients who completed a PR program in real-life settings.

Results: The estimated MCID for the 3-minute CRT and DIRECT scores was five repetitions and two units, respectively, using separate and combined independent anchors. The all-patient (body mass index-obstruction-dyspnea-exercise [BODE] scores 0-7), BODE 0-2 ( $n=42)$, and BODE 3-4 $(n=50)$ groups showed improvements greater than the MCID in most tests and questionnaires used. In contrast, the BODE 5-7 group $(n=24)$ showed improvements greater than MCID in only the 3-minute CRT, 6-minute walk test, endurance exercise test, and DIRECT questionnaire.

Discussion and conclusion: This study demonstrates that the short and simple DIRECT questionnaire and 3-minute CRT are responsive to capture the beneficial effects of a PR program in COPD patients, including those with severe disease.

Trial registration number: $\mathrm{NCT} 03286660$.

Keywords: MCID, chair tests, tools, COPD, pulmonary rehabilitation, outcomes assessment

\section{Introduction}

Pulmonary rehabilitation (PR) has not yet become the standard of care in real life for COPD patients despite a high level of evidence that it reduces dyspnea and improves health-related quality of life (HRQoL). ${ }^{1,2}$ While the low adoption rate can be attributed in part to regional differences in health care systems, geography, and PR prescriber/ patient preferences, ${ }^{3}$ one important aspect is the difficulty in assessing and understanding the results of a PR program by the referring physician himself, especially because assessment must be multidimensional. ${ }^{4}$

In clinical practice, the availability and/or feasibility of many PR outcome tests make them virtually inaccessible to a large proportion of practitioners. However, many simple tools are available that require no specialized equipment or facilities and can be used in routine medical practice. For example, the COPD Assessment Test $(\mathrm{CAT})^{5}$ contains only eight questions but is both simple to administer and sensitive to change, which contrasts with more complex questionnaires such as the 76-question St George's Respiratory Questionnaire (SGRQ). ${ }^{6}$ However, while the CAT may be 
multidimensional from a psychometric point of view, ${ }^{5}$ it does not accurately assess the impact of dyspnea on activities of daily life, nor does it capture improvements in functional capacity. In this regard, PR has proven efficacy in improving functional measures such as the distance covered during a 6-minute walk test (6MWT) or cyclo-ergometer endurance time. ${ }^{7}$ However, because these tests are often inaccessible for routine use, ${ }^{8}$ they cannot form the basis for evaluation in the less codified real-life environments where PR programs usually take place. To overcome these limitations, simple alternative field exercise tests ${ }^{9,10}$ have recently been evaluated in COPD patients and show promising results. Of particular interest are tests that do not require specialized equipment, such as the five-repetition sit-to-stand test (5STST), ${ }^{11}$ the 1-minute sit-to-stand test (1-minute STST), ${ }^{12}$ and the 3-minute chair rise test (3-minute CRT). ${ }^{13}$

In this study, we aimed to assess the minimal clinically important difference (MCID) change after PR of two validated additional simple tests: the Disability Related to COPD Tool (DIRECT), a short questionnaire focusing on the impact of dyspnea, ${ }^{14}$ and the 3-minute CRT, which yields physiological responses and symptoms that correlate closely with those of the $6 \mathrm{MWT} .{ }^{13}$ To optimize the external validity of the study, we conducted it as pragmatically as possible by 1) minimizing restrictions on disease severity for enrollment; 2) including varied contexts and modalities of PR, ranging from post-exacerbation inpatient hospital-based, outpatient hospital-based, and private physiotherapist-based programs; and 3) including diverse locations. Our goal was to explore what would be the most appropriate simple tests for a multidimensional evaluation of the benefits of a PR program in real-life settings.

\section{Methods}

This prospective multicenter observational study was conducted in real-life conditions with inpatient and outpatient populations from December 2015 to May 2017 (ClinicalTrials. gov: NCT03286660). The study was approved by the appropriate legal and ethical committees (CPP Nord Ouest IV decision no IDRBC: 2014A0085641). The patients received detailed information on the methods and objective of the study and provided written consent to participate. The study was conducted in accordance with the Declaration of Helsinki.

\section{Inclusion and exclusion criteria}

To preserve the pragmatic nature of the study, all patients with a diagnosis of COPD selected for PR by their attending physician were included if they had a $6 \mathrm{MWT}$ distance $>250 \mathrm{~m}$ upon enrollment. Exclusion criteria were minimized to the standard contraindications of PR (locomotor deficiencies and unstable cardiac conditions). Patients with COPD who suffered from additional known and significant respiratory conditions were not included in the study.

\section{PR programs}

At all locations, PR programs included upper/lower limb training and endurance (or aerobic) training on a cycloergometer and/or treadmill (all evaluations were performed with a cyclo-ergometer). Endurance training sessions were to be performed daily for inpatients and at least twice weekly for outpatients, with each training session targeting a minimum duration of 30 minutes.

\section{Measurements}

All patients were evaluated before initiation and at the end of the PR program.

\section{Standard measures}

Symptoms, health impairment status, and quality of life were assessed using the modified Medical Research Council (mMRC) dyspnea scale, ${ }^{15}$ the SGRQ,${ }^{6}$ the Hospital Anxiety Depression Scale (HADS), ${ }^{16}$ and the CAT. ${ }^{5}$ Exercise testing included 5STST, ${ }^{11}$ 1-minute STST, ${ }^{12}$ a $6 \mathrm{MWT}$ performed according to European Respiratory Society/American Thoracic Society guidelines, ${ }^{17}$ and an endurance cycle test performed at a work rate equal to $75 \%$ of maximal for each patient. Maximal work rate was determined during cardiopulmonary exercise testing within 3 months prior to or upon entry into the PR program. There was no specific order to complete all the questionnaires. There was only a recommendation to complete the SGRQ at a different time because of its length. Except for the different chair rise tests, there was no specific order to perform the exercise tests.

\section{Study-specific measures: DIRECT questionnaire}

The DIRECT ${ }^{14}$ questionnaire was administered at the same time as the mMRC, SGRQ, HADS, and CAT questionnaires. In brief, this questionnaire explores the frequency with which, "because of his/her shortness of breath", the patient has difficulties in performing certain daily tasks (six questions) or in engaging in relationships with others (four questions). A validated English version of the questionnaire is included in the supplementary material.

\section{Study-specific measures: chair rise tests}

After the chair rise tests (also known as sit-to-stand tests) were explained to the patients, they performed them in the order: 1) 5STST, ${ }^{11}$ 2) 1-minute STST, ${ }^{12}$ and 3) 3-minute CRT. ${ }^{13}$ Each 
exercise was separated by at least 20 minutes of rest, even if the heart rate and $\mathrm{SpO}_{2}$ had returned to baseline values before the end of 20 minutes. The tests were performed according to a standardized protocol with trained study staff, using a standard chair (height 46-48 cm) without armrests, stabilized against a wall. The patients sat with their knees and hips flexed to $90^{\circ}$, feet flat on the floor hip-width apart, and hands on the hips or chest. They were asked to stand up completely straight and to sit down landing firmly, without using their upper limbs to assist movement. Symptoms (Borg dyspnea and Borg fatigue scores) were evaluated before and at the end of each test following standard recommendations. ${ }^{18}$ Heart rate and $\mathrm{SpO}_{2}$ were recorded continuously from the start to the end of each session. Specific instructions for the conduct of the 3-minute CRT protocol are provided in the supplemental material.

\section{Statistical analysis}

Baseline characteristics are presented as mean \pm SD for normally distributed values and as median with interquartile range for nonnormal data and percentages. Between-group differences were tested with ANOVA for normally distributed variables and the Kruskal-Wallis test was used for non-Gaussian data. Categorical variables were tested with the chi-squared test when appropriate. Paired $t$-test and Wilcoxon signed rank test were used for normally and non-normally distributed data, respectively, when comparing paired measurements "before" and "after" PR. These data are presented as the mean change with $95 \%$ CIs.

In the realm of health care, a statistically significant difference may be of secondary importance to the health or quality of life of patients. To overcome these shortcomings, we have used the concept of an MCID which is another standard for determining effectiveness of a given treatment and describing patient satisfaction in reference to that treatment. MCIDs were estimated using anchor-based methods ${ }^{19}$ and distribution-based methods. The predefined criteria for establishing the validity of external anchors were a significant Pearson correlation (absolute value $>0.3$ ) between the change in score of the dependent variable (Y) and anchor variable (X). Since inference with Pearson's correlation assumes normally distributed score variables (or very large sample size), we dealt with non-normal data and possible outliers using the Donoho-Stahel estimator ${ }^{20}$ to compute a robust estimate of the correlation (termed robust correlation coefficient), which provides a more accurate estimate of the linear relationship between tested variables. As it is standard in anchor-based methods, MCIDs were estimated at the population level using linear regression to estimate the value of the $\mathrm{Y}$ variable corresponding to the minimum clinically important improvement in X. To justify the use of linear regression that models the relationship between the measured variable and the anchor as linear and not of other kind (which is sound for a MCID anchoring estimation), we have also estimated the relationship using a nonparametric analysis that does not pre-specify the function relating response $\mathrm{Y}$ to predictor $\mathrm{X}$ and only requires weak model assumptions, such as continuity and/or differentiability. We used a smoothing scatter plot (using cross-validation to select the smoothing parameter) with its confidence band. ${ }^{21}$ Linearity was considered valid when the nonparametric confidence band covered the legitimate linear regression. For each pair (Y, X), we also built receiver operating characteristic curves to assess the best Y score cut-off to discriminate between patients who improved by the established MCID for X, with equal weighting given to sensitivity and specificity (data not shown). Discrepancies between the individual scores of a standard and a robust fit were resolved by estimating the MCID of Y using a multiple linear regression on two variables (anchors) X1 and X2 with well-established MCID. Analyses and graph construction were conducted using $\mathrm{R}$ (version 3.4). A $P$-value of 0.05 was considered statistically significant.

\section{Results \\ Study population and baseline characteristics}

Of the 162 patients with COPD referred for a PR program, 149 were initially included in the study, and a total of 116 patients completed the program. The remaining 33 patients withdrew either because of an acute exacerbation $(n=9)$ or because the monitoring of the data sources revealed major methodological deviations in the performance of the tests. Obviously, this fact reveals the conditions of evaluations carried out in real-life conditions. Occasional data points concerning 6MWT, SGRQ, and endurance test were lacking mainly for practical reasons, but the amount of missing data concerning these scores did not justify omitting the corresponding patient records from the whole dataset for studying the observed population, which would result in much poorer sample sizes and loss of efficiency.

The study was conducted in nine centers and comprised four inpatient and five outpatient centers. Three inpatient centers enrolled 74 stable patients, 65 of whom completed the program; one inpatient center enrolled seven early postexacerbation patients, with five completing; two outpatient centers (private physiotherapists) enrolled 48 patients, with 41 completing; and three outpatient centers were day-care centers that enrolled 20 patients, with 16 completing the program. In total, the four inpatient centers provided 54\% and $55 \%$ of the total study population and fully evaluated patients, respectively, and the five outpatient centers provided 
Table I Baseline characteristics of patients stratified by BODE score

\begin{tabular}{|c|c|c|c|c|c|}
\hline Characteristics & $\begin{array}{l}\text { All patients, } \\
\mathbf{N}=116\end{array}$ & $\begin{array}{l}\text { BODE 0-2, } \\
N=42(36 \%)\end{array}$ & $\begin{array}{l}\text { BODE 3-4, } \\
N=50(43 \%)\end{array}$ & $\begin{array}{l}\text { BODE 5-7, } \\
\mathbf{N}=24(21 \%)\end{array}$ & $\begin{array}{l}\text { Overall } P \text {-value } \\
\text { (BODE } 0-2 \text { vs } 3-4 \\
\text { vs } 5-7 \text { ) }\end{array}$ \\
\hline Age (years) & $63.8 \pm 8.5$ & $62.3 \pm 7.5$ & $65.2 \pm 9.5$ & $63.3 \pm 7.8$ & 0.449 \\
\hline Sex, male $(n, \%)$ & $72(62.1)$ & $23(54.8)$ & $34(68.0)$ & $15(62.5)$ & 0.427 \\
\hline BMI $\left(\mathrm{kg} / \mathrm{m}^{2}\right)$ & $25.0 \pm 5.4$ & $27.0 \pm 5.0$ & $24.8 \pm 5.3$ & $21.9 \pm 5.0$ & $<0.00 \mathrm{I}^{\mathrm{a}}$ \\
\hline Smoking (pack-years) & $42.0 \pm 22.7$ & $38.7 \pm 15.9$ & $40.7 \pm 19.5$ & $50.9 \pm 35.6$ & 0.707 \\
\hline GOLD stage & & & & & $<0.00 \mathrm{I}^{\mathrm{a}}$ \\
\hline II & $49(42.2)$ & $36(85.7)$ & I3 (26.0) & $0(0.0)$ & \\
\hline III & $51(44.0)$ & $6(14.3)$ & $33(66.0)$ & $12(50.0)$ & \\
\hline IV & $16(13.8)$ & $0(0.0)$ & $4(8.0)$ & $12(50.0)$ & \\
\hline $\mathrm{FEV}_{1}(\mathrm{~L})$ & $1.3 \pm 0.5$ & $1.7 \pm 0.4$ & $1.2 \pm 0.3$ & $0.8 \pm 0.2$ & $<0.00 \mathrm{I}^{\mathrm{a}}$ \\
\hline $\mathrm{FEV}_{1} \%$ predicted (mean, $95 \% \mathrm{Cl}$ ) & $47.5(44.5-50.4)$ & $62.1(58.8-65.4)$ & $43.7(40.3-47.1)$ & $29.6(26.9-32.3)$ & $<0.001^{\mathrm{a}}$ \\
\hline $\mathrm{FEV}_{\mathrm{I}} / \mathrm{FVC} \%$ (mean, 95\% Cl) & $49.4(47.2-51.5)$ & $56.8(54.2-59.3)$ & $46.8(44.0-49.6)$ & $41.9(36.3-47.4)$ & $<0.00 \mathrm{I}^{\mathrm{a}}$ \\
\hline RV \% predicted (mean, $95 \% \mathrm{Cl}$ ) & $183.8(\mid 72.3-195.2)$ & $163.8(151.3-176.3)$ & I82.9 (I65.8-200.0) & $225.7(190.1-261.3)$ & $0.00 \mathrm{I}^{\mathrm{a}}$ \\
\hline RV/TLC \% predicted (mean, $95 \% \mathrm{Cl}$ ) & $152.3(140.6-164.0)$ & |36.1 (129.5-142.6) & $157.3(131.2-183.3)$ & $174.4(162.5-186.3)$ & $<0.00 \mathrm{I}^{\mathrm{a}}$ \\
\hline \multicolumn{6}{|l|}{ Maximal cycle test } \\
\hline Watts & $76.2 \pm 31.3$ & $96.4 \pm 27.6$ & $69.5 \pm 24.9$ & $47.1 \pm 23.7$ & $<0.00 \mathrm{I}^{\mathrm{a}}$ \\
\hline $\mathrm{VO}_{2}(\mathrm{~mL} / \mathrm{min} / \mathrm{kg})$ & $15.6 \pm 4.2$ & $17.8 \pm 4.1$ & $14.8 \pm 3.5$ & $12.4 \pm 3.4$ & $<0.001^{\mathrm{a}}$ \\
\hline Borg dyspnea score (median, QI, Q3) & $5.0(5.0,7.0)$ & $6.0(5.0,7.0)$ & $5.0(5.0,7.0)$ & $5.0(4.0,7.0)$ & 0.635 \\
\hline Borg fatigue score (median QI, Q3) & $7.0(4.0,7.0)$ & $7.0(5.0,7.5)$ & $6.5(5.0,7.0)$ & $5.0(2.0,6.0)$ & 0.478 \\
\hline $\mathrm{SpO}_{2}(\%)$ & $91.4 \pm 4.9$ & $93.4 \pm 3.7$ & $90.2 \pm 4.7$ & $89.6 \pm 6.3$ & $0.003^{\mathrm{a}}$ \\
\hline
\end{tabular}

Notes: aStatistically significant. Data are presented as the mean \pm SD unless indicated. None of the patients scored in the BODE 8-I0 point range.

Abbreviations: $\mathrm{BODE}$, body mass index-obstruction-dyspnea-exercise; $\mathrm{BMI}$, body mass index; $\mathrm{RV}$, residual volume; $\mathrm{TLC}$, total lung capacity; $\mathrm{VO}_{2}$, oxygen uptake; $\mathrm{SpO}_{2}$, oxygen saturation.

the balance. Baseline characteristics for the patients classified by BODE score ${ }^{22}$ are described in Table 1 . The mean $(95 \% \mathrm{CI})$ number of sessions completed was: all patients $(\mathrm{n}=116), 43.2$ (38.0-48.4); BODE 0-2 group $(\mathrm{n}=42), 50.2$ (40.1-60.3); BODE 3-4 group ( $\mathrm{n}=50)$, 41.7 (34.1-49.3); and BODE 5-7 group ( $\mathrm{n}=24), 34.9$ (25.9-44.0).

\section{Assessment of MCID for DIRECT and 3-minute CRT}

Of the 116 patients who completed the PR program, 88 patients had a complete dataset from all evaluations at both the beginning and end of the program and were included in the determination of MCID for the DIRECT questionnaire and 3-minute CRT. Even with the reduced dataset, highly significant robust Pearson correlations were obtained

Table 2 Anchor-based estimates of the minimal clinically important differences for the 3-min CRT and DIRECT questionnaire

\begin{tabular}{l|l|l}
\hline Tool & Anchor & MCID (95\% CI) \\
\hline DIRECT & CAT & $-1.7 I(-2.26 ;-I .17)^{\mathrm{b}}$ \\
& I-min STST & $-0.92(-1.92 ; 0.086)$ \\
\multirow{3}{*}{$3-m i n$ CRT } & CAT +I-min STST & $-1.27(-2.57 ; 0.02)$ \\
& I-min STST & $3.91(1.53 ; 6.28)^{\mathrm{c}}$ \\
& SGRQ & $5.73(3.79 ; 7.68)$ \\
& SGRQ + I-min STST & $4.97(2.07 ; 7.88)$ \\
\hline
\end{tabular}

Notes: aAll patients ( $n=88)$; ${ }^{\mathrm{a}}$ questionnaire score units; ' repetitions.

Abbreviations: I-min STST, I minute sit-to-stand test; 3-min CRT, 3 minute chair rise test; CAT, COPD assessment test; CRT, chair rise test; MCID, minimal clinically important difference; SGRQ, St. George's Respiratory Questionnaire. between DIRECT and CAT/1-minute STST and between 3-minute CRT and SGRQ/1-minute STST (Table S1). For each dependent variable, we determined a MCID of $>1$ unit for the DIRECT score and five repetitions for the 3-minute CRT with separate and combined independent anchors (Tables 2 and $\underline{\text { S2). }}$. As one can notice in Table 3, the MCIDs determined by distribution-based methods are of the same order. So, taking into account these complementary statistical estimations, we propose a MCID of $\geq 2$ units for the DIRECT score and $\geq 5$ repetitions for the 3 -minute CRT.

\section{Effects of PR on patient-related outcomes and exercise variables}

Table 4 shows the baseline variables and their changes after PR (detailed results are given in Table S3). As expected, the

Table 3 Distribution-based method for the estimation of the minimal clinically important differences for the 3-minute CRT and DIRECT questionnaire

\begin{tabular}{l|l|l}
\hline Tool & Statistical methods & MCID $(95 \% \text { CI })^{\mathrm{a}}$ \\
\hline DIRECT & Cohen's effect size & $-1.97(-2.3 \mathrm{I} ;-\mathrm{I} .7 \mathrm{I})^{\mathrm{b}}$ \\
& SEM & $-2.78(-3.27 ;-2.43)$ \\
& Empirical rule & $-1.89(-2.22 ;-1.65)$ \\
\multirow{3}{*}{-minute CRT } & Cohen's effect size & $4.69(4.09 ; 5.5 \mathrm{I})^{\mathrm{c}}$ \\
& SEM & $4.33(3.77 ; 5.09)$ \\
& Empirical rule & $4.5 \mathrm{I}(3.93 ; 5.29)$ \\
\hline
\end{tabular}

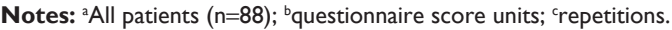

Abbreviations: CRT, chair rise test; SEM, standard error of measurement; MCID, minimal clinically important difference; DIRECT, Disability Related to COPD Tool. 


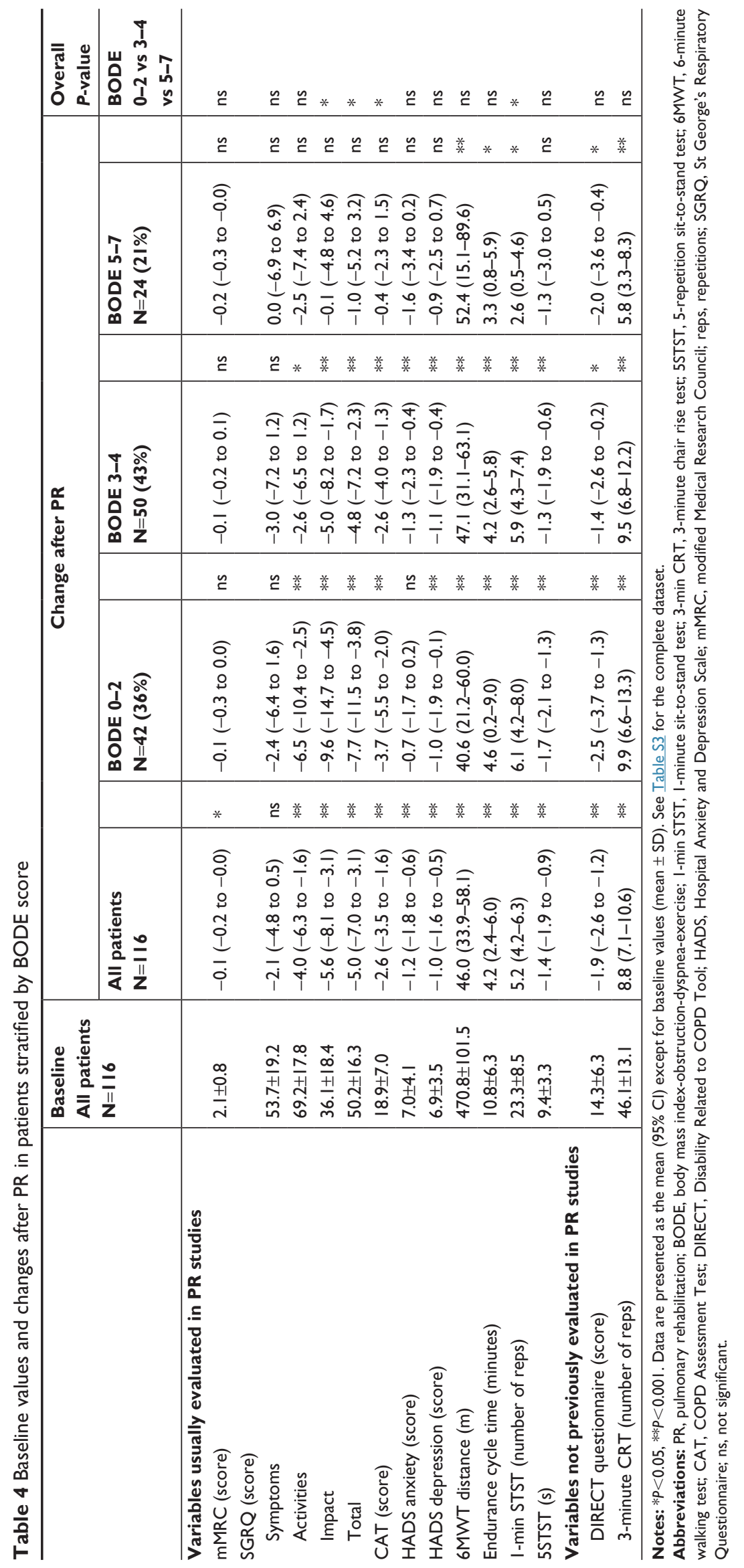


entire population as well as the BODE 0-2 and 3-4 groups showed significant improvement above their respective MCIDs in the majority of exercise tests and questionnaires, the exceptions being 5STST, mMRC, HADS, and SGRQ symptoms. ${ }^{23-27}$ In contrast, the BODE 5-7 group showed significant improvement for the DIRECT questionnaire, 6MWT, 1-minute STST, ${ }^{12,28}$ endurance test, and 3-minute CRT. Of these, all except the 1-minute STST achieved improvements above the MCIDs. The overall $P$-values were significant only for the change in 1-minute STST, CAT, and SGRQ impact and total scores, indicating that the effects of PR on these, but not other, variables were dependent on the BODE categories.

\section{Comparison between I-minute STST and 3-minute CRT}

Figure 1 shows the mean number of repetitions performed during the 1-minute STST and each minute of the 3-minute CRT according to the BODE categories. The first minute of the 3-minute CRT was capped at 20 or 12 repetitions (see descriptions in the supplementary material); therefore, the mean number of repetitions was significantly lower during the first minute of the 3-minute CRT than during the 1-minute STST (15.6 \pm 3.7 vs 23.3 \pm 8.5 , respectively). Because of the different test durations, the total number of repetitions was higher and the alterations in Borg dyspnea and fatigue scores and $\mathrm{SpO}_{2}$ were larger during the 3-minute CRT than during the 1-minute STST (Figure 1 and Table S3).

\section{Discussion}

The main contribution of this study is to determine, in routine practice, the MCID after PR of a short questionnaire focusing on the impact of dyspnea (DIRECT) ${ }^{14}$ and the 3-minute CRT, which yields physiological responses and symptoms that correlate closely with those of the $6 \mathrm{MWT} .{ }^{13}$ In this study, real-life conditions were achieved by enrolling patients in different states of COPD (ranging from stable to early post-exacerbation), using PR programs that were not standardized between centers, and employing different settings (hospital inpatients and outpatients, practitioners' offices). The results confirm that PR is able to improve exercise capacity and patient-centered outcomes in COPD patients regardless of disease severity, and also that the 3-minute CRT and DIRECT questionnaire are short and simple tools that are validated for assessing these outcomes.

Performing a study in which neither the PR setting nor the PR program was normalized carried with it some risk. However, our results are consistent with the published literature

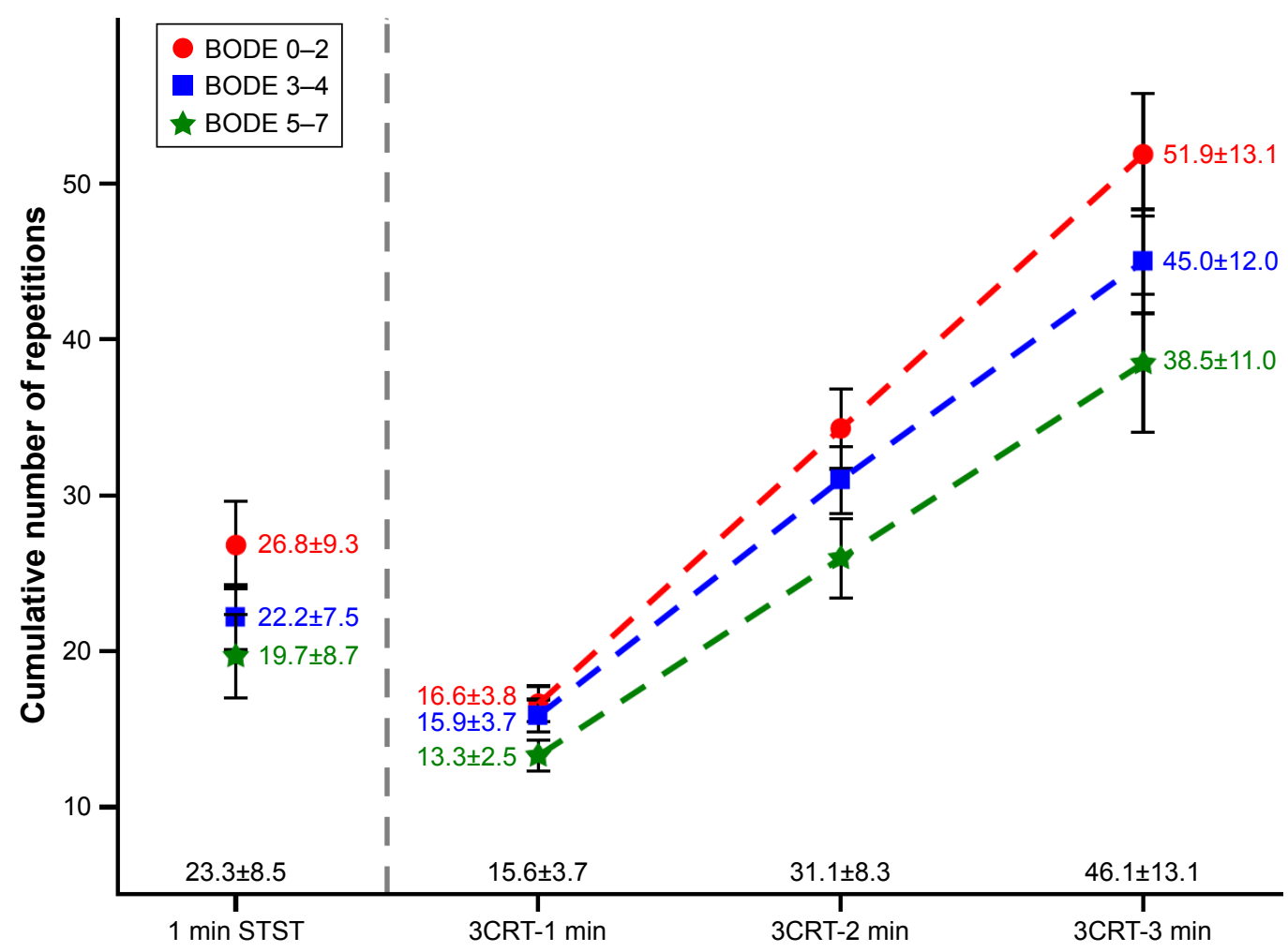

Figure I Cumulative number of repetitions during the I-minute STST and 3-minute CRT.

Notes: COPD patients were stratified by BODE score ( $0-2, n=42 ; 3-4, n=50 ; 5-7, n=24)$ and the number of repetitions (mean \pm SD) during the I-minute STST and 3-minute CRT was calculated. The numbers on the $x$-axis in black represent the mean \pm SD values for the entire patient cohort $(n=116)$.

Abbreviations: BODE, body mass index-obstruction-dyspnea-exercise; I-min STST, I-minute sit-to-stand test; 3CRT, 3-minute chair rise test. 
in showing PR-related improvements in symptoms, HRQoL, and functional exercise capacity assessed by 5STST, 1-minute STST, 3-minute CRT, 6MWT, and endurance tests. ${ }^{1,2,4}$ In general, the improvements observed in our study were not strongly dependent on the disease severity, although some indicators (mMRC, SGRQ, CAT, 5STST, and 1-minute STST) did not change significantly in the most severely ill patients. Wedzicha et $\mathrm{al}^{29}$ found that patients with MRC 3-4, but not those with MRC 5, improved their shuttle walk test and HRQoL scores after PR, as assessed by SGRQ and Chronic Respiratory Disease questionnaires. Recently, Sciriha et $\mathrm{al}^{30}$ also found that PR could improve the CAT and SGRQ scores of COPD patients with mMRC 3-4. No doubt, the results of these studies might have been different had the mMRC4 patients been analyzed separately. Indeed, our choice to stratify the patients according to the BODE index, which is a more detailed reflection of disease severity than the mMRC or MRC, may explain the difference in results between studies. At the same time, our results may not fully reflect real life because the most severely ill patient group was small $(n=24)$. Nevertheless, our study reinforces the clinical robustness of $\mathrm{PR}$ in COPD regardless of the modalities of intervention, as recently suggested by Spruit et al. ${ }^{31}$

This study provides the first data analysis comparing the physiological and symptomatic responses of three simple sitto-stand tests and longer tests, such as the 6MWT and endurance test, in the context of a PR program. Our results confirm that short exercise tests, particularly the 3-minute CRT, are as responsive as the longer tests; indeed, the 3-minute CRT yielded results very similar to those obtained with the 6MWT in an earlier proof-of-concept study. ${ }^{13}$ This feature may be advantageous for both the PR prescriber and the patient, since the magnitude of the improvement from the 1-minute STST ( $\sim 6$ repetitions) and 3-minute CRT ( $\sim 10$ repetitions) allows them to more readily and objectively perceive the benefits of PR, at least for the BODE 0-4 category patients. Our indepth comparison of responses to the 1-minute STST and 3-minute CRT suggests that, although the 1-minute STST is useful for exploring exercise responses, longer duration tests provide additional information. For example, maximal oxygen desaturation, heart rate, and Borg symptoms were similar during the 3-minute CRT and 6MWT but differed from those during the 1-minute STST. Moreover, our finding that the 3-minute CRT improvement after PR is not affected by the BODE category (in contrast to the 1-minute STST) suggests that it could be equally useful for every patient, regardless of disease severity. However, this assumption needs to be validated with a larger population. Finally, this study allowed us to identify a reliable MCID of about five repetitions for the 3-minute CRT using different anchors. Furthermore, the dual anchoring on functional exercise capacity and HRQoL increases the importance of the 3-minute CRT as a tool to assess the global benefit of PR.

The results of this study also reveal the responsiveness of the DIRECT questionnaire to PR. This is an important finding because this short questionnaire focusing on the impact of dyspnea on daily activities offers a significant advantage over the mMRC, ${ }^{25}$ for example, which we confirm was not responsive to $\mathrm{PR}$. We also verified that the MCID of the CAT (with SGRQ as anchor) was similar in our cohort $(-1.90 ; 95 \% \mathrm{CI}-3.09$ to -0.72$)$ to that obtained with a larger cohort of patients who benefited from a PR program $(\mathrm{n}=565$ : $-2.5 ; 95 \% \mathrm{CI}-3.0$ to -1.9$) .{ }^{27}$ Notably, our study is the first to establish an MCID for the DIRECT questionnaire, which we estimated at two units based on a combined anchor method and distribution methods. In contrast to the DIRECT questionnaire and exercise tests, improvement in the CAT score did not reach the MCID for the BODE 5-7 group in our study, although CAT was previously shown to be responsive to PR for COPD patients in all mMRC classes. ${ }^{24,30,32}$ This difference could be due to our patient stratification by BODE score, which, to the best of our knowledge, has not been used before. As was the case for the 3-minute CRT, we found that PR improved the DIRECT scores independently of disease severity. Therefore, the CAT and DIRECT questionnaires appear to have complementary utility for assessing the shortterm benefit of PR; in contrast, the change in HADS scores did not reach their known MCIDs. ${ }^{26}$

Finally, it can be considered that the relatively small number of patients for a multicenter study is a limitation for the external validity of the study. However, despite the heterogeneity of patients and centers, the MCID determinations are statistically robust and are also legitimized by the use of our collaborators for several years.

In conclusion, this pragmatic study mainly determines the MCID of DIRECT and 3-minute CRT after PR. Moreover, these results argue that short questionnaires (CAT and DIRECT) and simple chair rise tests (1-minute STST and 3-minute CRT) are able to capture multiple complementary dimensions of the benefits of a PR program for a large cohort of COPD patients with varying disease severity. Future studies should determine whether a multidimensional analysis with simple tools could be used to track the diminishing benefits of PR over time, a particularly important question in this field.

\section{Data sharing statement}

The authors intend to share the entire individual participant data collected during the trial after deidentification. They also 
intend to share the study protocol and informed consent form. The data will be accessible in electronic format immediately following publication for 5 years.

\section{Acknowledgment}

The authors would like to thank Anne M O'Rourke, PhD, for editing the manuscript.

\section{Disclosure}

Financial support for this study was provided by an unrestricted grant from Boehringer Ingelheim, France. BA reports grants and other from Boehringer Ingelheim, France, during the conduct of the study. The authors report no other conflicts of interest in this work.

\section{References}

1. Spruit MA, Singh SJ, Garvey C, et al. An official American Thoracic Society/European Respiratory Society statement: key concepts and advances in pulmonary rehabilitation. Am J Respir Crit Care Med. 2013;188(8):e13-e64.

2. McCarthy B, Casey D, Devane D, et al. Pulmonary rehabilitation for chronic obstructive pulmonary disease. In: The Cochrane Collaboration, editor. Cochrane Database of Systematic Reviews [Internet]. Chichester, UK: John Wiley \& Sons, Ltd; 2015 [cited 2017 Jul 25]. Available from: http://doi.wiley.com/10.1002/14651858.CD003793.pub3. Accessed January 4, 2018.

3. Rochester CL, Vogiatzis I, Holland AE, et al. An Official American Thoracic Society/European Respiratory Society Policy Statement: Enhancing Implementation, Use, and Delivery of Pulmonary Rehabilitation. Am J Respir Crit Care Med. 2015;192(11):1373-1386.

4. Spruit MA, Augustin IM, Vanfleteren LE, et al. Differential response to pulmonary rehabilitation in COPD: multidimensional profiling. Eur Respir J. 2015;46(6):1625-1635.

5. Jones PW, Harding G, Berry P, Wiklund I, Chen WH, Kline Leidy N. Development and first validation of the COPD Assessment Test. Eur Respir J. 2009;34(3):648-654.

6. Jones PW, Quirk FH, Baveystock CM. Littlejohns P. A self-complete measure of health status for chronic airflow limitation. Am Rev Respir Dis. 1992;145(6):1321-1327.

7. Puente-Maestu L, Palange P, Casaburi R, et al. Use of exercise testing in the evaluation of interventional efficacy: an official ERS statement. Eur Respir J. 2016;47(2):429-460.

8. Kocks JW, Asijee GM, Tsiligianni IG, Kerstjens HA, van der Molen T. Functional status measurement in COPD: a review of available methods and their feasibility in primary care. Prim Care Respir J. 2011;20(3): 269-275.

9. Lee AL, Harrison SL, Beauchamp MK, Janaudis-Ferreira T, Brooks D. Alternative field exercise tests for people with respiratory conditions. Curr Phys Med Rehabil Rep. 2015;3(3):232-241.

10. Maddocks M, Nolan CM, Man WD. Simple functional tests in COPD: stand up and be counted!. Eur Respiratory Soc. 2017;49:1700104.

11. Jones SE, Kon SS, Canavan JL, et al. The five-repetition sit-to-stand test as a functional outcome measure in COPD. Thorax. 2013;68(11): 1015-1020.

12. Crook S, Büsching G, Schultz K, et al. A multicentre validation of the 1-min sit-to-stand test in patients with COPD. Eur Respir J. 2017;49(3): 1601871.

13. Aguilaniu RH, Jondot M. A simple semipaced 3-minute chair rise test for routine exercise tolerance testing in COPD. Int J Chron Obstruct Pulmon Dis. 2014;9:1009-1019.
14. Aguilaniu B, Gonzalez-Bermejo J, Regnault A, et al. Disability related to COPD tool (DIRECT): towards an assessment of COPD-related disability in routine practice. Int J Chron Obstruct Pulmon Dis. 2011;6:387-398.

15. Bestall JC, Paul EA, Garrod R, Garnham R, Jones PW, Wedzicha JA. Usefulness of the Medical Research Council (MRC) dyspnoea scale as a measure of disability in patients with chronic obstructive pulmonary disease. Thorax. 1999;54(7):581-586.

16. Snaith RP. The Hospital Anxiety And Depression Scale. Health Qual Life Outcomes. 2003;1:29.

17. Holland AE, Spruit MA, Troosters T, et al. An official European Respiratory Society/American Thoracic Society technical standard: field walking tests in chronic respiratory disease. Eur Respir J. 2014;44(6): 1428-1446.

18. Borg GA. Psychophysical bases of perceived exertion. Med Sci Sports Exerc. 1982;14(5):377-381.

19. Revicki D, Hays RD, Cella D, Sloan J. Recommended methods for determining responsiveness and minimally important differences for patient-reported outcomes. J Clin Epidemiol. 2008;61(2):102-109.

20. Maronna RA, Yohai VJ. The Behavior of the Stahel-Donoho Robust Multivariate Estimator. J Am Stat Assoc. 1995;90(429):330-341.

21. Kadafar K. Applied Smoothing Techniques for Data Analysis: The Kernel Approach with S-PLUS Illustrations [Internet]. Journal of the American Statistical Association 1999; [cited 2018 Jan 16]. Available from: http://link.galegroup.com/apps/doc/A57472170/ AONE?sid=googlescholar. Accessed January 4, 2018.

22. Celli BR, Cote CG, Marin JM, et al. The body-mass index, airflow obstruction, dyspnea, and exercise capacity index in chronic obstructive pulmonary disease. $N$ Engl J Med. 2004;350(10):1005-1012.

23. Borel B, Provencher S, Saey D, Maltais F. Responsiveness of Various Exercise-Testing Protocols to Therapeutic Interventions in COPD. Pulm Med. 2013;2013(4):1-11.

24. Smid DE, Franssen FM, Houben-Wilke S, et al. Responsiveness and MCID Estimates for CAT, CCQ, and HADS in Patients With COPD Undergoing Pulmonary Rehabilitation: A Prospective Analysis. J Am Med Dir Assoc. 2017;18(1):53-58.

25. Crisafulli E, Clini EM. Measures of dyspnea in pulmonary rehabilitation. Multidiscip Respir Med. 2010;5(3):202-210.

26. Puhan MA, Frey M, Büchi S, Schünemann HJ. The minimal important difference of the hospital anxiety and depression scale in patients with chronic obstructive pulmonary disease. Health Qual Life Outcomes. 2008;6(1):46.

27. Kon SS, Canavan JL, Jones SE, et al. Minimum clinically important difference for the COPD Assessment Test: a prospective analysis. Lancet Respir Med. 2014;2(3):195-203.

28. Vaidya T, de Bisschop C, Beaumont M, et al. Is the 1-minute sit-tostand test a good tool for the evaluation of the impact of pulmonary rehabilitation? Determination of the minimal important difference in COPD. Int J Chron Obstruct Pulmon Dis. 2016;11:2609-2616.

29. Wedzicha JA, Bestall JC, Garrod R, Garnham R, Paul EA, Jones PW. Randomized controlled trial of pulmonary rehabilitation in severe chronic obstructive pulmonary disease patients, stratified with the MRC dyspnoea scale. Eur Respir J. 1998;12(2):363-369.

30. Sciriha A, Lungaro-Mifsud S, Scerri J, Magro R, Camilleri L, Montefort S. Health status of COPD patients undergoing pulmonary rehabilitation: a comparative responsiveness of the CAT and SGRQ. Chron Respir Dis. 2017;14(4):352-359.

31. Spruit MA, Pitta F, Garvey C, et al. Differences in content and organisational aspects of pulmonary rehabilitation programmes. Eur Respir J. 2014;43(5):1326-1337.

32. Kon SS, Clark AL, Dilaver D, et al. Response of the COPD Assessment Test to pulmonary rehabilitation in unselected chronic respiratory disease. Respirology. 2013;18(6):974-977. 
International Journal of COPD

\section{Publish your work in this journal}

The International Journal of COPD is an international, peer-reviewed journal of therapeutics and pharmacology focusing on concise rapid reporting of clinical studies and reviews in COPD. Special focus is given to the pathophysiological processes underlying the disease, intervention programs, patient focused education, and self management protocols.

\section{Dovepress}

This journal is indexed on PubMed Central, MedLine and CAS. The manuscript management system is completely online and includes a very quick and fair peer-review system, which is all easy to use. Visit $\mathrm{http}: / / \mathrm{www}$.dovepress.com/testimonials.php to read real quotes from published authors.

Submit your manuscript here: http://www.dovepress.com/international-journal-of-chronic-obstructive-pulmonary-disease-journal 